\title{
Pierre BRECHON, Frédéric GONTHIER et \\ Sandrine ASTOR (dir.), La France des valeurs. \\ Quarante ans d'évolutions
}

\section{Gaël Brulé}

\section{(2) OpenEdition}

Journals

Édition électronique

URL : http://journals.openedition.org/ress/5457

DOI : $10.4000 /$ ress.5457

ISBN : 1663-4446

ISSN : $1663-4446$

Éditeur

Librairie Droz

Édition imprimée

Date de publication : 20 décembre 2019

Pagination : 316-319

ISSN : 0048-8046

Référence électronique

Gaël Brulé, « Pierre BRECHON, Frédéric GONTHIER et Sandrine ASTOR (dir.), La France des valeurs.

Quarante ans d'évolutions », Revue européenne des sciences sociales [En ligne], 57-2 | 2019, mis en ligne le 20 décembre 2019, consulté le 01 décembre 2020. URL : http://journals.openedition.org/ress/5457 ; DOI : https://doi.org/10.4000/ress.5457

Ce document a été généré automatiquement le 1 décembre 2020

(c) Librairie Droz 


\title{
Pierre BRECHON, Frédéric GONTHIER et Sandrine ASTOR (dir.), La France des valeurs. Quarante ans d'évolutions
}

\author{
Gaël Brulé
}

\section{RÉFÉRENCE}

Pierre BRECHON, Frédéric GONTHIER et Sandrine ASTOR (dir.), 2019, La France des valeurs. Quarante ans d'évolutions, Grenoble, Presses universitaires de Grenoble, 381 p.

1 La France des valeurs, coordonné par Pierre Bréchon, Frédéric Gonthier et Sandrine Astor, se compose de 57 chapitres courts (4 à 6 pages) répartis sur huit grandes parties. Il explore les thèmes suivants: appartenances sociales et identités individuelles, nouvelles formes du lien social, morale individuelle et normes collectives, familles en mutation, valeurs économiques et sens du travail, religion en mouvement, politique en crise, politiques publiques sous tension. L'ouvrage s'appuie principalement sur l'enquête de référence de valeurs des Européens, le European Values Study, qui comprend cinq vagues depuis les années 1970, ainsi que sur des enquêtes ponctuelles comme l'Eurobaromètre ou l'enquête Valeurs de 2018.

2 La première partie concerne les appartenances sociales et les identités individuelles. On y constate des tendances assez attendues, comme la reproduction des inégalités par milieu social, la diversification anthropologique de la population (augmentation du nombre de personnes dont au moins un parent est né à l'étranger), l'assouplissement de la norme du mariage, l'émergence de formes plus souples de cohabitation ainsi que l'augmentation du célibat. Mais l'on y découvre des résultats moins intuitifs, comme la hausse de l'intérêt pour la lecture, la politisation accrue des individus ou encore l'augmentation marquée du sentiment d'appartenance à la France (le pourcentage de 
personnes déclarant être très fières d'être françaises est passé de $35 \%$ en 1981 à $51 \%$ en 2018).

3 La deuxième partie traite des liens de confiance et de méfiance, et du bonheur des Français. On y retrouve un schéma connu dans la société française : une forte confiance dans les cercles familiaux et une forte défiance envers ceux que l'on ne connait pas. Cependant l'intolérance à l'égard de groupes religieux et culturels minoritaires est en baisse, notamment en ce qui concerne les musulmans (le pourcentage de répondants déclarant ne pas vouloir de musulman comme voisin est passé de $18 \%$ en 1990 à $8 \%$ à 2018). Par ailleurs, il apparaît que le niveau de bonheur ou de satisfaction à l'égard de sa vie n'a fait qu'augmenter en 30 ans. L'engagement associatif est à la hausse, notamment chez les populations les plus éduquées, en raison peut-être de la diminution des ressources mobilisables par l'État, qui entraîne un remplacement des solidarités froides par des solidarités chaudes. Cette tendance est aussi à référer à un altruisme plus prononcé chez les jeunes générations.

4 La troisième partie s'intéresse à la morale individuelle et aux normes collectives. Elle décrit une libéralisation des mœurs, c'est-à-dire une tolérance croissante par rapport à des questions comme l'homosexualité, l'avortement, le divorce, l'euthanasie, le suicide, l'aventure extraconjugale ou la prostitution. De manière assez surprenante, on observe un phénomène de « convergence générationnelle » : si l'on isole la classe d'âge 70 ans et plus, il n'y a guère de différences significatives entre les générations. Par contre, et cela confirme les précédentes études, le libéralisme est plus important chez les gens plus éduqués, de catégories socio-professionnelles supérieures et positionnés à gauche. Paradoxalement, l'on observe plutôt une évolution vers une revendication de davantage d'ordre et d'autorité, et ce dans toutes les classes d'âge.

5 La quatrième partie, familles en mutation, interroge les opinions autour de questions comme le mariage, les enfants, la répartition des rôles. Sur ces sujets, on semble osciller entre rémanence des valeurs et changements sociétaux rapides. Par exemple, la progression du mariage comme « institution dépassée » n'a que peu évolué entre 1981 (29\% de personnes interrogées sont d'accord) et 2018 (35\%). En revanche, la progression du divorce comme justifié a plus que doublé dans la même période (63\% des interrogés expriment leur accord en 2018 contre $31 \%$ en 1981). Les facteurs considérés comme importants pour le succès du mariage ont peu évolué, à l'exception de l'item «avoir un revenu convenable » qui a baissé de 41 à $29 \%$. La fidélité reste le facteur le plus important ( $83 \%$ en 2018 contre $72 \%$ en 1981), particulièrement revendiqué par les plus jeunes, les moins diplômés, et au sein des milieux religieux. Au niveau de la répartition des rôles, on observe un rééquilibrage fort, notamment chez les hommes qui voient de plus en plus comme légitime le partage des tâches familiales et un accès équitable à l'emploi. Les enfants sont de moins en moins vus comme un élément obligatoire et structurant du couple. Par ailleurs les qualités attendues chez eux changent : la persévérance, la générosité et l'indépendance sont de plus en plus valorisées, l'obéissance de moins en moins.

6 La cinquième partie est dédiée aux valeurs économiques et au sens donné au travail. On observe sur quatre vagues d'enquête une « désaffection à l'égard du marché » qui se stabilise. Le libéralisme économique est plus prononcé chez les plus riches, les gens de droite et les plus politisés. La confiance accordée aux grandes entreprises et aux syndicats, assez faible dans les deux cas (environ 42 et $37 \%$ ), clive les populations de droite, plus proches des entreprises, et de gauche, plus proches des syndicats. 
L'importance accordée au travail, toujours très élevée, semble néanmoins être relativisée par les populations plus jeunes. On constate également une diminution du jugement négatif émis à l'égard de celles et ceux qui n'ont pas de travail dans l'ensemble de la population ( $33 \%$ des personnes interrogées estimaient que les chômeurs devraient être obligés d'accepter toute offre d'emploi en 2008 contre 28 \% en 2018).

7 La sixième partie traite du fait religieux. On y découvre sans réelle surprise une chute libre du nombre de Français catholiques fréquentant un office religieux ou déclarant croire en Dieu, tandis que la tendance est inverse chez les musulmans, et chez les protestants. À partir d'un indice de libéralisme des mœurs (les répondants déclarent justifiés ou non des comportements comme l'homosexualité, l'euthanasie, le suicide, les relations sexuelles hors mariage, la prostitution), les musulmans apparaissent moins réceptifs en moyenne à la liberté des mœurs que les chrétiens et les non-affiliés (les personnes se déclarant sans religion) : $52 \%$ des musulmans interrogés se situent en dessous de la valeur 5 sur une échelle de 1 (moins tolérant) à 10 (plus tolérant), contre $20 \%$ des chrétiens et $10 \%$ des non affiliés.

8 La septième partie s'enquiert des questions politiques. On y voit un clivage gauchedroite solidement ancré, et une grande stabilité de l'intérêt des citoyens pour la politique, très loin du phénomène de désintéressement souvent déploré par les éditorialistes. Si certaines institutions (l'Église, les partis politiques) suscitent moins de confiance qu'auparavant (ce qui n'est pas le cas néanmoins de la santé, de l'armée, de la police), la demande politique semble au contraire en hausse et la volonté de participer de plus en plus forte. Elle est simplement plus diversifiée : les formes d'action politique hors des scrutins se développent, par le biais de pétitions, de boycotts et de manifestations.

9 La huitième et dernière partie est dédiée aux politiques publiques et à des grandes questions comme l'identité nationale, la place du gouvernement et l'environnement. L'Union Européenne divise la population française, puisque $47 \%$ des répondants déclarent lui faire "plutôt confiance" (notamment les personnes interrogées appartenant aux catégories professionnelles supérieures) tandis que $47 \%$ des répondants ne lui font "plutôt pas confiance" (particulièrement les jeunes, les personnes appartenant aux catégories sociales inférieures, et les sympathisants de l'extrême-gauche et de l'extrême-droite). La proportion de gens prêts à prendre les armes pour défendre son pays est de $66 \%$ en 2018, soit le plus haut score relevé depuis le début l'enquête ( $42 \%$ en 1981). La question des droits donnés au gouvernement français pour intervenir sur les questions de terrorisme divise, notamment sur la possibilité ou non d'accéder aux informations personnelles ( $27 \%$ des sympathisants de la France Insoumise se déclarent absolument contre la vidéosurveillance contre $6 \%$ des sympathisants de la République en Marche et des Républicains) Enfin, si les répondants déclarent que l'environnement est plus important que la croissance, ils se mobilisent inégalement pour l'environnement, les plus éduqués étant les plus actifs.

10 En raison de la diversité des thèmes couverts, l'objectif de La France des valeurs est ambitieux. Considérant le nombre de chapitres, un cadre unificateur était nécessaire. C'est ce que proposent Pierre Bréchon, Frédéric Gonthier et Sandrine Astor dans le chapitre de conclusion. Loin d'une crise des valeurs et d'un changement radical souvent déplorés, les coordinateurs du volume montrent au contraire que les valeurs présentent une grande inertie. Au lieu d'une vague d'individualisme qui balayerait 
toute forme de solidarité sur son passage, on observe plutôt des logiques de solidarité qui évoluent peu, dans le sens d'ailleurs de la pensée postmoderne qui voit davantage une recomposition qu'un délitement des formes de solidarités. Les valeurs de libéralisme des mœurs et de tolérance sont à la hausse, même si, comme les auteurs le rappellent de manière salutaire, les réponses données aux enquêtes ne se traduisent pas toujours en réalité puisque la tolérance revendiquée envers les populations gitanes ou les personnes en proie à l'addiction (alcool ou drogues) se traduit par des stratégies d'évitement dans les faits. L'ouvrage pointe aussi des évolutions de fond. Ainsi, le clivage hommes-femmes semble s'effacer peu à peu et les inégalités sont de moins en moins bien perçues, notamment chez les hommes.

11 On peut parfois se poser la question de la causalité mise en avant par les auteurs. Par exemple, affirmer que le « positionnement politique est le déterminant-clé de plusieurs attitudes » est certainement vrai d'un point de vue corrélationnel, mais l'on pourrait également émettre l'hypothèse que ce sont au contraire les attitudes qui préconditionnent les positionnements politiques. Est-ce que parce que je suis de gauche, je valorise une certaine égalité des chances ou est-ce que mon appétence pour l'égalité me fait tendre vers la gauche de l'échiquier?

12 Néanmoins La France des valeurs réussit son pari de présenter une encyclopédie des valeurs et livre ce faisant une étude extensive des valeurs des Français. Les auteurs ont su dépeindre un paysage qui, très loin d'une "crise des valeurs » souvent clamée, présente une grande viscosité.

\section{AUTEURS}

\section{GAËL BRULÉ}

Université de Neuchâtel - Université de Genève 